\title{
Pitot probe corrections in fully developed turbulent pipe flow
}

\author{
B J McKeon, J Li ${ }^{1}$, W Jiang ${ }^{2}$, J F Morrison ${ }^{3}$ and A J Smits \\ Department of Mechanical and Aerospace Engineering, Princeton University, Princeton, \\ NJ 08544-0710, USA
}

Received 14 April 2003, in final form and accepted for publication

19 June 2003

Published 21 July 2003

Online at stacks.iop.org/MST/14/1449

\begin{abstract}
Mean flow measurements taken in fully developed turbulent pipe flow over a wide Reynolds number range are used to evaluate current methods of correcting Pitot probe data. Based on this evaluation, a new form for the displacement correction is proposed which appears to be more accurate over a wider range of conditions than those currently available. The difficulty of obtaining the true near-wall velocity profile near the wall is explored.
\end{abstract}

Keywords: fluid flow velocity corrections, Pitot probe, displacement correction, calibration

(Some figures in this article are in colour only in the electronic version)

$\begin{array}{ll}\text { Nomenclature } \\ B & \text { Log law constant } \\ C_{\mathrm{p}} & \text { Measured pressure coefficient } \frac{p_{0}-p_{\text {meas }}}{0.5 \rho U^{2}} \\ d & \text { Pitot probe diameter } \\ d^{+} & \text {Non-dimensional Pitot diameter } \frac{d u_{\tau}}{v} \\ E & \text { Fractional difference between measured veloc- } \\ & \text { ity and Spalding's relationship } \\ K_{\mathrm{T}} & \text { Directional sensitivity of Pitot probe } \\ \operatorname{Re}_{\mathrm{d}} & \frac{d U_{\text {local }}}{v} \\ \operatorname{Re}_{\mathrm{D}} & \text { Pipe Reynolds number, based on average } \\ \overline{u^{2}}, \overline{v^{2}}, \overline{w^{2}} & \text { velocity, } \bar{U}, \text { and pipe diameter, } D \\ & \text { Mean square of velocity fluctuations in } x, r \text { and } \\ U & \theta \text { directions } \\ U^{+} & \text {True local mean velocity } \\ U_{\mathrm{m}} & \frac{U}{u_{\tau}} \\ u_{\tau} & \text { Measured mean velocity } \\ X^{*} & \text { Friction velocity } \sqrt{\tau / \rho} \\ & \text { Patel's Preston probe non-dimensional parame- } \\ & \text { ter log } \frac{\Delta p_{\mathrm{p}} D^{2}}{4 \rho v^{2}}\end{array}$

1 Permanent address: School of the Built Environment, Victoria University of Technology, PO Box 14428, MCMC, Melbourne, Australia.

2 Permanent address: CARDC, PO Box 211 Mianyang, Sichuan 621000, People's Republic of China.

3 Permanent address: Department of Aeronautics, Imperial College, London SW7 2BY, UK.
$Y^{*} \quad$ Patel's Preston probe non-dimensional parameter $\log _{10} \frac{\tau_{0} D^{2}}{4 \rho v^{2}}$

$y \quad$ Distance from the wall

$y_{\mathrm{c}} \quad$ Distance from the wall to probe centreline

$y^{+} \quad$ Non-dimensional distance from the wall $\frac{y u_{\tau}}{v}$

$\alpha \quad$ Non-dimensional velocity gradient (equation (5))

$\beta \quad$ Non-dimensional second derivative of velocity (equation (6))

$\Delta y \quad$ Displacement of streamlines due to presence of probe

$\delta_{\mathrm{w}} \quad$ Displacement in the wall region

$\Delta p_{\mathrm{p}} \quad$ Difference between pressure measured by Pitot or Preston probe and undisturbed static pressure von Kármán constant

$\kappa$
$\rho$$\quad$ Fluid kinematic viscosity

$\rho \quad$ Fluid density

$\tau_{\mathrm{w}} \quad$ Wall shear stress

\section{Summary}

To obtain accurate mean velocity profiles using a Pitot probe, corrections need to be made for the effects of viscosity, turbulence, velocity gradient and the presence of a wall. Recent measurements by Zagarola and Smits (1998) (referred to from now on as ZS) in turbulent pipe flow over Reynolds numbers ranging from $31 \times 10^{3}$ to $35 \times 10^{6}$ have raised 


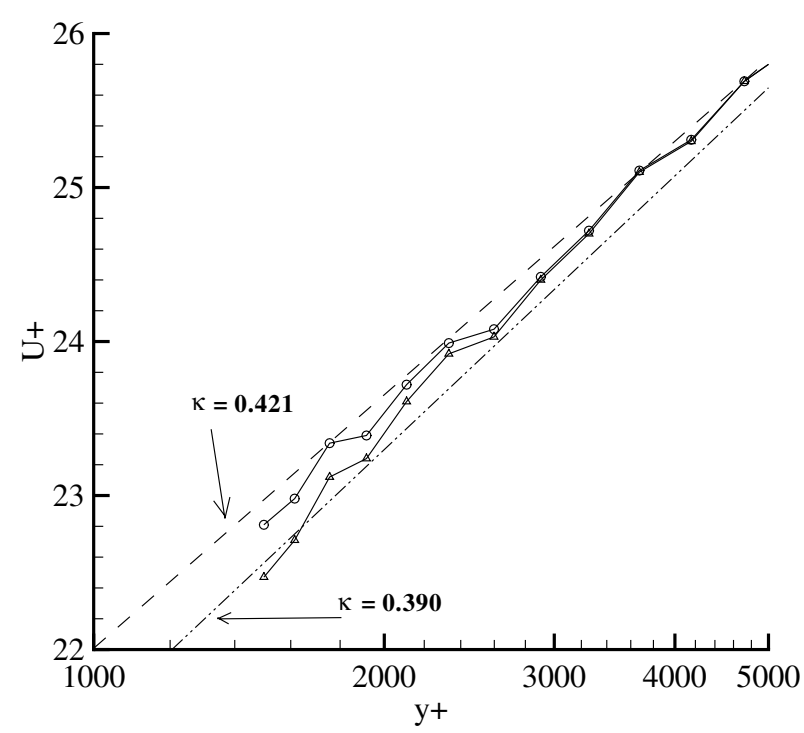

Figure 1. Effect of application of a wall correction term to estimated slope of the overlap region for data corrected using the MacMillan (1954) displacement correction: $\triangle$, no wall term, MacMillan displacement correction; $\bigcirc$, MacMillan wall term and displacement corrections.

some questions regarding the accuracy and applicability of the current correction methods (see, for example, Perry et al 2001). To illustrate the importance of these corrections, we find that at a pipe flow Reynolds number of $6 \times 10^{6}$ there is a difference of greater than $5 \%$ in the slope of the logarithmic region between data with and without a wall correction (figure 1). This is important given the ongoing debate concerning the existence and slope of the log law (e.g. ZS 1998, Barenblatt and Chorin 1998) and the popular use of the slope of the velocity profile in the near-wall region to determine the von Kármán constant.

\section{Introduction}

\subsection{Viscous correction}

Viscous corrections are important when the Reynolds number based on probe diameter, $R e_{\mathrm{d}}$, is less than about 1000 . Using the results obtained by Barker (1922), Hurd et al (1953), MacMillan (1954, 1956), and Chue (1975), Zagarola (1996) suggested that for $R e_{\mathrm{d}}>30$ :

$$
C_{\mathrm{p}}=1+\frac{10}{R e_{\mathrm{d}}^{1.5}}
$$

where $C_{\mathrm{p}}$ is the measured pressure coefficient. For all the results presented here, $R e_{\mathrm{d}}$ was always greater than 100 and the viscous correction never exceeded the maximum value of $0.5 \%$ of the local mean velocity observed at the lowest pipe Reynolds number. For the present purposes we will therefore assume that equation (1) is a true representation of viscous effects on Pitot probe data.

\subsection{Turbulence correction}

The effects of turbulence on the Pitot tube reading are twofold. First, the velocity fluctuations increase the measured total pressure. Second, when measurements are taken using a

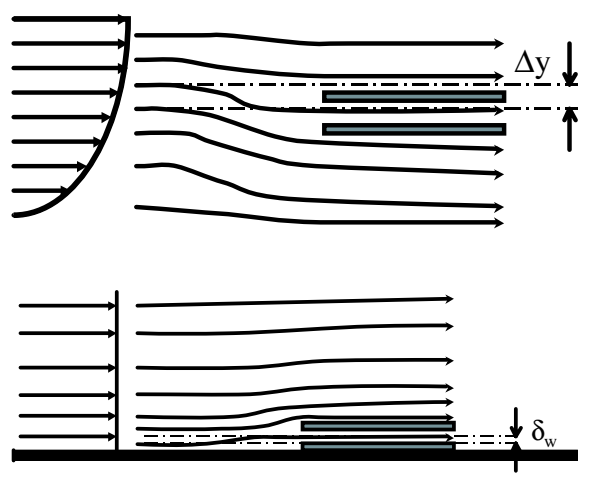

Figure 2. The effect of a Pitot probe on the streamline pattern: top in a shear flow and bottom in uniform flow near a wall.

pressure tapping at the wall to measure the static pressure, the radial gradient in static pressure due to velocity fluctuations must be taken into account. The combined effect of these two phenomena is given by (Ozarapoglu 1972)

$$
\frac{U-U_{\mathrm{m}}}{U_{\mathrm{m}}}=-\frac{\overline{u^{2}}-\left(1+K_{\mathrm{t}}\right) \overline{v^{2}}+\left(1-K_{\mathrm{t}}\right) \overline{w^{2}}}{2 U_{\mathrm{m}}^{2}} .
$$

Here $U$ is the true mean velocity, $U_{\mathrm{m}}$ is the measured mean velocity, $\overline{u^{2}}, \overline{v^{2}}$ and $\overline{w^{2}}$ are the mean square velocity fluctuations in the streamwise, radial and circumferential directions respectively, and $K_{\mathrm{t}}$ is a coefficient that accounts for the directional sensitivity of the Pitot probe and is approximately equal to 0.3 .

Dickinson (1975) suggested that for $y^{+}>50$ equation (2) could be represented more simply by

$$
\frac{U-U_{\mathrm{m}}}{U_{\mathrm{m}}}=-\frac{\overline{u^{2}}}{2 U_{\mathrm{m}}^{2}},
$$

although this should perhaps be reviewed in the light of the Reynolds number dependence of the anisotropy of the Reynolds stress tensor (which is, of course, not well understood). Equation (3) was used by ZS to correct their Pitot probe data for the effects of turbulence, and we will use the same correction where appropriate.

\subsection{Velocity gradient correction}

In a shear flow, the presence of the probe deflects the streamlines so that the probe registers a velocity that is higher than the velocity at the geometric centre of the probe (figure 2, top). To account for this interference effect, which usually dominates all other Pitot probe errors, a correction is made which can be expressed as an error in the measured velocity, or as an apparent shift in the probe location.

If the velocity variation across the Pitot tube is small compared to its mean value, and if we assume that the mean velocity is a function of $y$ only, then we can write

$$
\frac{\Delta U}{U\left(y_{\mathrm{c}}\right)}=\frac{U\left(y_{\mathrm{c}}+\Delta y\right)-U\left(y_{\mathrm{c}}\right)}{U\left(y_{\mathrm{c}}\right)}=2 \alpha \frac{\Delta y}{d}-\beta \frac{(\Delta y)^{2}}{d^{2}}+\cdots
$$

where $y_{\mathrm{c}}$ is the position of the centre of the Pitot tube, $U\left(y_{\mathrm{c}}\right)$ is the true velocity at the centre, $\Delta y$ is the correction in position due to streamline displacement and the velocity gradients are 
evaluated at the geometric centre of the probe. $\alpha$ and $\beta$ are defined by:

$$
\begin{gathered}
\alpha=\left.\frac{d}{2 U\left(y_{\mathrm{c}}\right)} \frac{\mathrm{d} U}{\mathrm{~d} y}\right|_{\mathrm{c}} \\
\beta=-\left.\frac{1}{2} \frac{d^{2}}{U\left(y_{\mathrm{c}}\right)} \frac{\mathrm{d}^{2} U}{\mathrm{~d} y^{2}}\right|_{\mathrm{c}} .
\end{gathered}
$$

Equation (4) shows that the correction in velocity and the apparent shift of probe position $\Delta y / d$ are directly connected. In fact, the velocity gradient correction is usually implemented by correcting the probe position rather than by correcting the velocity itself. This form of the correction is usually called the displacement correction, and several authors (MacMillan 1954, Livesey 1956, Patel 1965, Ozarapoglu 1972, Tavoularis and Szymczak 1989) have suggested that

$$
\frac{\Delta y}{d}=\varepsilon
$$

where $0.08<\varepsilon<0.16$. When $\varepsilon=0.15$, it is called the MacMillan correction; this is the most widely used correction for the velocity gradient error.

Another popular choice is the correction proposed by Chue (1975), who used Patel's (1965) data to argue that the displacement correction should depend on the local velocity gradient. Zagarola (1996) proposed that Chue's displacement correction can be represented by the curve-fit

$$
\frac{\Delta y}{d}=0.18 \alpha\left(1-0.17 \alpha^{2}\right)
$$

and ZS applied this displacement correction to all their results According to equation (8), $\Delta y / d$ approaches a constant value of 0.15 as $\alpha \rightarrow 1$ (near the wall), and goes to zero as $\alpha \rightarrow 0$ (as it should, for example on the centreline of a pipe). In contrast, equation (7) leads to a discontinuity at the pipe centreline. Young and Maas (1936) raised this issue, but Tavoularis and Scymczak argued that there is not a problem if the correction is viewed in velocity terms and that, in general, the case where $\alpha=0$ is of no practical interest because it corresponds to a case with vanishing error. Equations (7) and (8) are displayed in figure 3 .

In the presence of a wall, there is an additional mechanism for streamline displacement. Consider a Pitot probe resting on the wall in uniform flow (figure 2, bottom). Since it resembles a forward-facing step, it may be expected that the streamlines would be displaced away from the wall, that is, towards the region of higher velocity (rather than towards the region of lower velocity as it is in free shear). Therefore the true correction close to the wall must be some combination of these two effects. MacMillan observed this trend and suggested that a wall correction was necessary in addition to the displacement correction for $y / d<2$. He proposed that this wall correction may be represented by

$$
\frac{\Delta U}{U}=0.015 \exp \left[-3.5\left(\frac{y}{d}-0.5\right)\right] .
$$

It should be noted that the data used to obtain the Chue and MacMillan corrections were not corrected for the effects of turbulence, and therefore both corrections combine the effects of turbulence intensity and velocity gradient. In other words, implementing an additional turbulence correction when using the Chue and MacMillan methods is not appropriate.

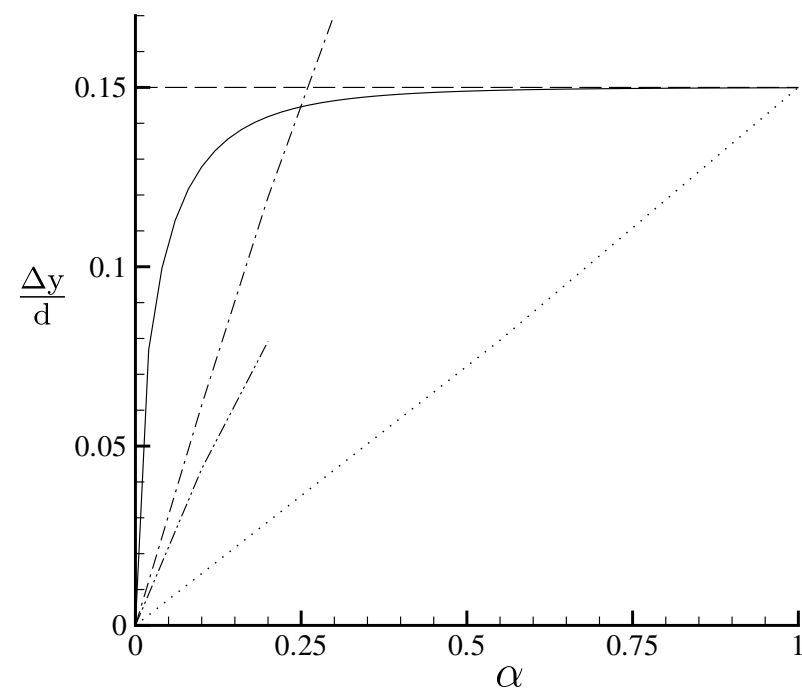

Figure 3. - - - MacMillan correction; $\cdots$... Chue correction _ - - - analytical form for spherical probes derived by Hall (1956); — - - analytical form for spherical probes derived by Lighthill (1957); — new correction.

\section{Experiment}

Pitot probes of four different diameters were used to measure mean velocity profiles in fully developed turbulent pipe flow in the Princeton Superpipe apparatus (see Zagarola (1996) for a full description). The Pitot tubes had outer diameters of 0.30 , $0.51,0.90$ and $1.83 \mathrm{~mm}$, with an inner to outer diameter ratio of 0.6. The pressure tapping correction of McKeon and Smits (2002) was applied to the static pressure measurements for all Reynolds numbers. A single normal constant-temperature hot-wire probe was also used, employing a $2.5 \mu \mathrm{m}$ diameter tungsten wire with an active length of $0.5 \mathrm{~mm}$, mounted on a Dantec P01 body and powered by a Dantec M10 series anemometer.

Two pipe flow Reynolds numbers were studied using all four probes: $75 \times 10^{3}$ and $146 \times 10^{3}$. In addition, 21 mean velocity profiles were obtained using the $0.3 \mathrm{~mm}$ diameter Pitot probe covering Reynolds numbers from $75 \times 10^{3}$ to $35 \times 10^{6}$. These profiles were compared with the ZS data taken with a $0.9 \mathrm{~mm}$ OD probe for Reynolds numbers from $31 \times 10^{3}$ to $35 \times 10^{6}$. The data points cover the range $d^{+}>10$ and $430<D / d<720$

All the Pitot probe data presented here were corrected for viscous effects using equation (1). Where appropriate, the correction for the effects of turbulence was applied using equation (3), using the turbulence intensity levels obtained by Morrison et al (2002).

The experimental apparatus and techniques used in the present study were for the most part the same as those used by ZS, and their error analysis applies almost unchanged to the measurements reported here. However, to improve the accuracy of the wall distance measurement, a new positioning system incorporating a DC motor and a linear encoder with an accuracy of $5 \mu \mathrm{m}$ per count was used to determine the wall distance $y$ (ZS reported an accuracy of $25 \mu \mathrm{m}$ ). In addition, the starting position for the Pitot probe was determined by detecting the electrical contact between the Pitot probe and the pipe surface to an accuracy of $5 \mu \mathrm{m}$, compared to $50 \mu \mathrm{m}$ for ZS. 
The accumulated position error of running the present traverse system forward and backward once over a distance of $71 \mathrm{~mm}$ was generally less than $30 \mu \mathrm{m}$, compared with $50 \mu \mathrm{m}$ accuracy for ZS. The position accuracy was estimated to be $\pm 1.7 \%$ for measuring points close to the pipe surface and $\pm 0.05 \%$ for data points taken near the centre of the pipe. Across the pipe radius, 57 data points were taken with logarithmically uniform spacing. The first point was that closest to the pipe surface (with the Pitot probe touching the pipe surface), the 56th point was at the centre of the pipe, and the 57th point was on the other side of the pipe centre. The experimental data were sampled using a PC-based data acquisition system at $500 \mathrm{~Hz}$ over a 2 minute period.

\section{Proposed correction}

\subsection{Displacement correction}

We now propose a new form of the velocity gradient correction, given by:

$$
\frac{\Delta y}{d}=0.15 \tanh (4 \sqrt{\alpha}) .
$$

This form is based on the analysis by Hall (1956) and Lighthill (1957) who found that the displacement correction for a sphere in a velocity gradient could be expressed as a hyperbolic tangent function of the non-dimensional shear. The evidence supporting equation (10) is presented in section 5. The constants in this equation were chosen to give the best collapse of the Pitot tube data considered here over the largest range of Reynolds number. Note that the new correction gives zero displacement for zero shear and asymptotes to the MacMillan correction for large shear $(\alpha \rightarrow 1)$. The new correction is shown in figure 3 along with the analytical forms derived by Hall (1956) and Lighthill (1957).

\subsection{Wall correction}

The new wall correction is based on the Preston probe data of Patel (1965). A Preston probe is simply a Pitot tube resting on the wall, a condition where the probe is under the maximum influence of the wall as well as shear. Patel's Preston probe curves are as follows:

(a) For $0<Y^{*}<1.5$ and $d^{+}<11$,

$$
Y^{*}=0.037+0.5 X^{*} \text {. }
$$

(b) For $3.5<Y^{*}<5.3$ and $110<d^{+}<1600$,

$$
X^{*}=Y^{*}+2.0 \log \left(1.95 Y^{*}+4.10\right) \text {. }
$$

Here

$$
X^{*}=\log _{10} \frac{\Delta p_{\mathrm{p}} d^{2}}{4 \rho v^{2}} \quad \text { and } \quad Y^{*}=\log _{10} \frac{\tau_{\mathrm{w}} d^{2}}{4 \rho v^{2}}
$$

where $\Delta p_{\mathrm{p}}$ is the Preston probe dynamic pressure, $d^{+}=$ $d u_{\tau} / \nu, u_{\tau}=\sqrt{\tau_{\mathrm{w}} / \rho}, \tau_{\mathrm{w}}$ is the wall shear stress, and $\rho$ and $v$ are the fluid density and kinematic viscosity respectively.

If we assume that the Preston tube reads the true pressure at

$$
y=0.5 d\left(1+\frac{\delta_{\mathrm{w}}}{d}\right)
$$

we have a definition of the wall displacement correction $\delta_{\mathrm{w}}$. When the form of the velocity profile is known, $\Delta p_{\mathrm{p}}=\frac{1}{2} \rho U^{2}$ may be formulated and substituted into the expression for $X^{*}$. The resulting expressions may then be compared with Patel's Preston probe curves to obtain $\delta_{\mathrm{w}}$ in each region. For example, equation (11) may be matched in the viscous sublayer (for $\left.y^{+}<4\right)$ to the accepted form of the profile:

$$
U^{+}=y^{+}
$$

where $U^{+}=U / u_{\tau}$ and $y^{+}=y u_{\tau} / \nu$. In addition, equation (12) may be matched (for $y^{+}>55$ ) to an approximate logarithmic region given by

$$
U^{+}=\frac{1}{\kappa} \ln y^{+}+B .
$$

The values $\kappa=0.418$ and $B=5.45$ were suggested by Patel, and are retained here for the sake of consistency.

For $8<d^{+}<110$, viscous effects are undoubtedly important, and we know that $\alpha$ changes significantly, so that we expect that $\delta_{\mathrm{w}}$ will vary with $d^{+}$. It was found, however, that a constant value of $\delta_{\mathrm{w}}$ in this region fits our data well. Hence we obtain the new wall correction (applicable for $y / d<2$ ):

$$
\frac{\delta_{\mathrm{w}}}{d}= \begin{cases}0.150 & \text { for } d^{+}<8 \\ 0.120 & \text { for } 8<d^{+}<110 \\ 0.085 & \text { for } 110<d^{+}<1600 .\end{cases}
$$

In this formulation, the wall displacement correction $\delta_{\mathrm{w}}$ is applied for increasing $y^{+}$until $y / d<2$, at which point the free shear displacement correction given by equation (10) is applied instead. Note that MacMillan also proposed a wall displacement term for $y / d<2$ but this was meant to be used in addition to the displacement correction for free shear.

\section{Evaluation of displacement and wall corrections}

To evaluate displacement and wall corrections, it would be convenient if the 'true' velocity profile was known. The proper correction is then the one that collapses all the Pitot tube data on the true profile, independent of Pitot tube size. Unfortunately, the nature of the true profile is still a source of debate, specifically as to what role geometry and/or Reynolds number play, particularly in the buffer region. However, even in the absence of a known profile, we can judge the accuracy of the correction by how well it collapses data taken with different diameter Pitot probes, over the full Reynolds number range. Here, we adopt this criterion as the primary standard for judging the accuracy of correction methods.

\subsection{Applicability of an additional turbulence correction}

We begin by determining whether or not an additional correction for turbulence intensity is required for each correction.

Figure 4 shows the effect of different corrections on the 0.3 and $0.9 \mathrm{~mm}$ data sets with no explicit turbulence correction. The MacMillan and new corrections collapse the data well, and the corrected data blend well into the established $u^{+}=y^{+}$ scaling near the wall. The Chue correction, however, does not collapse the data to the same degree. 


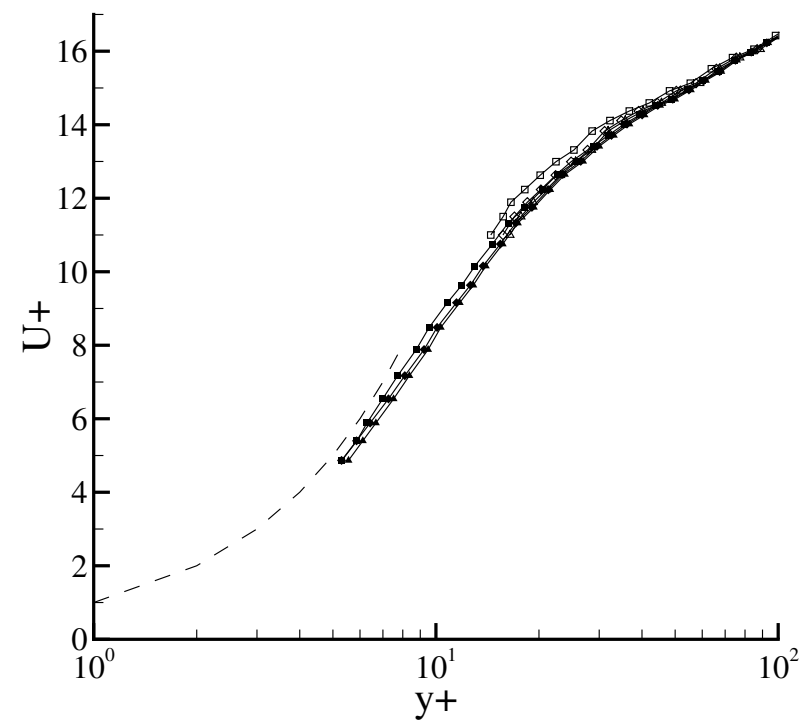

Figure 4. The effect of each displacement correction for $R e_{\mathrm{D}}=75 \times 10^{3}: \square$, Chue; $\triangle$, MacMillan; $\diamond$, new corrections. Filled symbols, $0.3 \mathrm{~mm}$ probe; open symbols, $0.9 \mathrm{~mm}$ probe. $--U^{+}=y^{+}$.

As was indicated earlier, it is not appropriate to make a turbulence correction in addition to the Chue, MacMillan and new corrections since the effects of turbulence are already implicitly accounted for in these methods. Nevertheless, it is often done in practice (see, for example Perry et al 2001). To see the effects of this procedure, the turbulence correction given by equation (3) was applied to all data, in addition to the Chue, MacMillan and new displacement corrections. It is apparent from figure 5 that the Chue correction still does not collapse the different data sets. In addition, the MacMillan and new corrections are now much too large to allow the data to blend into $u^{+}=y^{+}$.

Based on the poor performance of the Chue method, with and without a turbulence correction, this correction will not be examined further in this study. The failure of the Chue correction is important in that it was the correction method used by ZS. The ZS data have been reanalysed using the new correction, incorporating at the same time a new static pressure correction developed by McKeon and Smits (2002), and they were reported by McKeon et al (2003).

Note that the effects of turbulence intensity on the reading of total pressure compete with the effects of turbulence in defining the radial pressure gradient since they are of opposite sign. Applying both corrections at $R e_{\mathrm{D}}=7 \times 10^{6}$ and $y^{+}=4000$ changes $u^{+}$by only $0.2 \%$, despite the high value of $\frac{\left(\bar{u}^{2}\right)^{2}}{\left(2 U_{m}^{2}\right)^{2}}(>6)$. Therefore it appears that there is not a significant error introduced by the lack of a separate turbulence correction for the MacMillan and new corrections.

\subsection{Corrections in the overlap region}

As a first diagnostic for the displacement and wall corrections, the profiles are examined for Reynolds numbers where the points closest to the wall already lie in the overlap region, that is, for $R e_{\mathrm{D}}>10^{6}$ (McKeon et al 2003). Since the corrections themselves are small, and it is difficult to distinguish the even

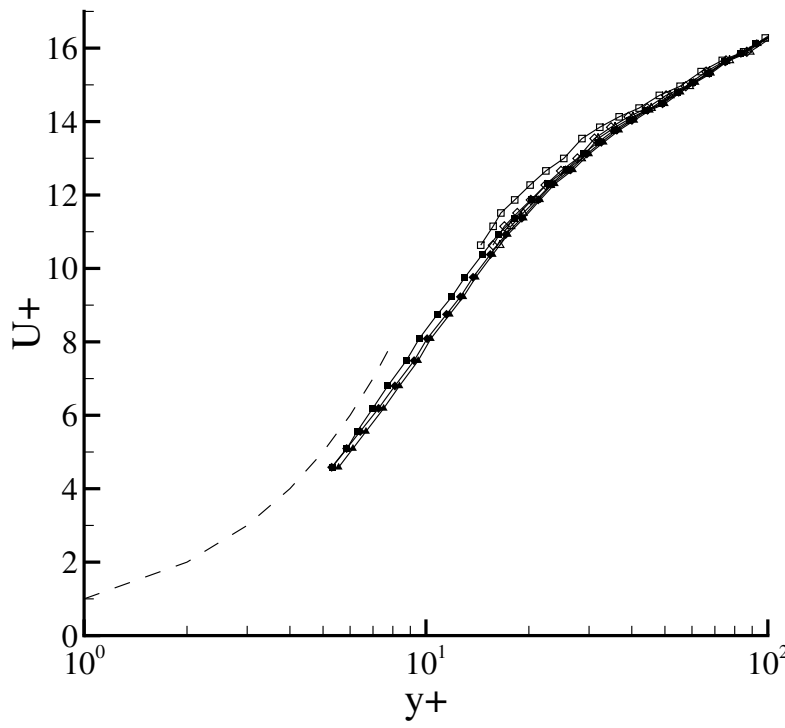

Figure 5. The effect of applying an explicit turbulence correction as well as a displacement correction, $R e_{\mathrm{D}}=75 \times 10^{3}$. Symbols as for figure 4

smaller differences between them, it is convenient to examine the deviation, $E$, of the corrected profile from Spalding's (1961) 'universal' profile, where

$$
E=\frac{y_{\mathrm{corr}}^{+}-y_{\mathrm{sp}}^{+}}{y_{\mathrm{sp}}^{+}} .
$$

Spalding's profile is an analytical representation of the profile for wall-normal positions up to and including the overlap layer. It is given by

$$
y^{+}=u^{+}+\mathrm{e}^{-\kappa B}\left(\mathrm{e}^{\kappa u^{+}}-1-\kappa u^{+}-\frac{\left(\kappa u^{+}\right)^{2}}{2}-\frac{\left(\kappa u^{+}\right)^{3}}{6}\right)
$$

and it was derived by curve-fitting data taken from pipes and boundary layers. The constants $\kappa=0.421$ and $B=5.60$, as proposed by McKeon et al (2003), will be used here. It is not suggested here that Spalding's profile is an accurate representation of the near-wall velocity profile in pipe flow (indeed we will show that it is not), only that it provides a convenient reference curve for purposes of comparison.

An important property of a displacement correction must be that it collapses data taken at the same Reynolds number with different diameter probes. Figures 6-8 show the variation of $E$ for each correction and data from both diameters of probe in the region $y / R<0.1$ and $y^{+}>100$ for selected Reynolds numbers greater than $10^{6}$.

Figure 6 shows clearly that both the MacMillan and new displacement corrections fail near the wall in the absence of a wall term. Data taken with different diameters do not collapse within approximately two diameters of the wall. Figure 7 shows that almost identical results are obtained for both corrections if we only use data points for $y>2 d$, that is, beyond where the wall terms would be applied. This was also the conclusion of McKeon et al (2003), who found consistent results concerning the range of existence of the log law and log law constants, independent of the displacement correction used, for data with $y \geqslant 2 d$. 

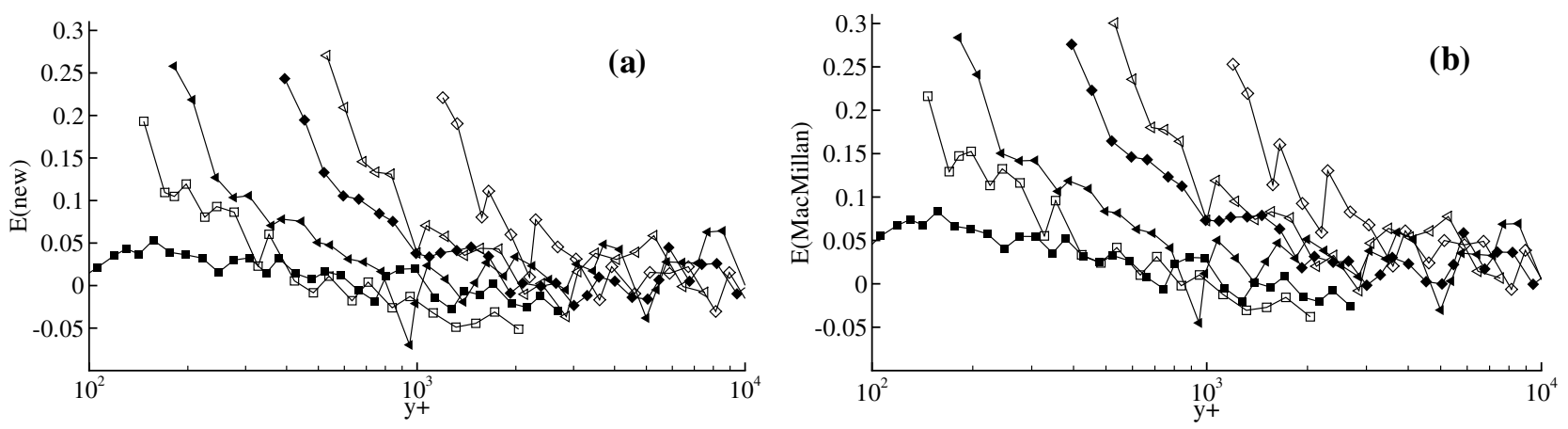

Figure 6. Deviation from the Spalding correlation for selected $R e_{\mathrm{D}} \geqslant 10^{6}$, new (a) and MacMillan (b) corrections with no wall corrections, all data points used. Solid symbols $0.3 \mathrm{~mm}$ Pitot, open symbols $0.9 \mathrm{~mm}$ Pitot.
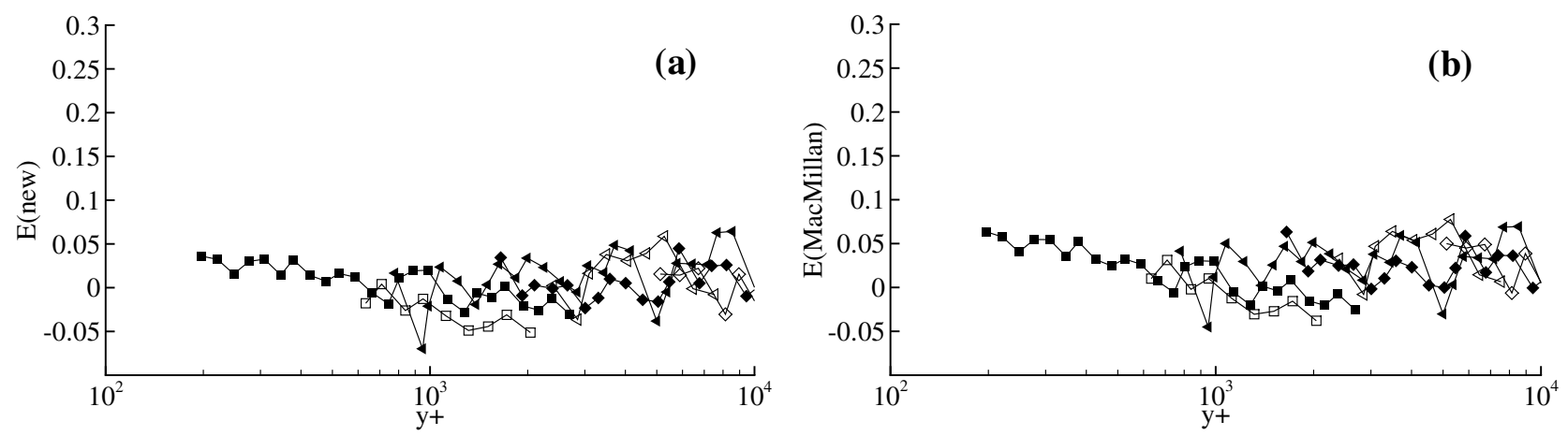

Figure 7. Deviation from the Spalding correlation for selected $R e_{\mathrm{D}} \geqslant 10^{6}$, new (a) and MacMillan (b) corrections with no wall corrections, points for $y>2 d$ only. Symbols as in figure 6 .
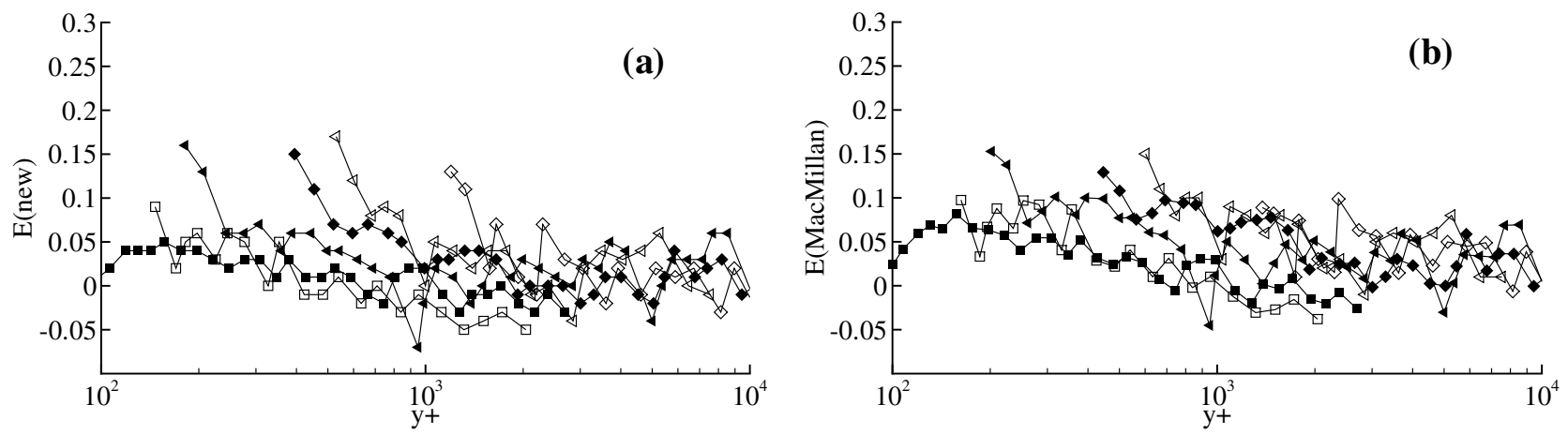

Figure 8. Deviation from the Spalding correlation for selected $R e_{\mathrm{D}} \geqslant 10^{6}$, new (a) and MacMillan (b) corrections with wall terms, all data points used. Symbols as in figure 6.

In figure 8, the MacMillan and new wall terms have been applied in addition to the displacement corrections of figures 6 and 7. It can be seen that in both cases the collapse with diameter is much improved, but there are still difficulties near the wall. For $y<1 d$, the new correction does not give as good a collapse as for the MacMillan correction but for $1 d<y<2 d$ the situation is reversed. This point is illustrated in figure 9 where points with $y<1 d$ have been removed from the data set. In this case, the new correction leads to full collapse with diameter while the MacMillan correction does not.

It should be noted that collapse of the profiles with Reynolds number has not been used as a criterion for evaluation of the corrections, but McKeon et al (2003) have shown that the profiles collapse to a single curve independent of Reynolds number, at least for the Reynolds numbers considered here.
If the displacement corrections are reformulated to show the percentage change in velocity and not position, as per equation (4), two observations may be made. First, the second term in the expansion for $\Delta U / U$, the ' $\beta$ ' term in equation (4), is small for all data presented here, and it can be neglected in our analysis. Second, the definition of $\alpha$ (equation (5)) may be written as

$$
\alpha=\left.\frac{d^{+}}{2 U^{+}\left(y_{\mathrm{c}}\right)} \frac{\mathrm{d} U^{+}}{\mathrm{d} y^{+}}\right|_{\mathrm{c}}
$$

which shows the Reynolds number dependence of $\alpha$ through the $d^{+}$term. It is clear that $\alpha$ will vary considerably for the points closest to the wall, where the apparent $\mathrm{d} U^{+} / \mathrm{d} y^{+}$is large. Young and Maas (1936), for example, noted that a constant displacement correction will only be valid if the gradient of velocity across the Pitot tube is approximately constant. 

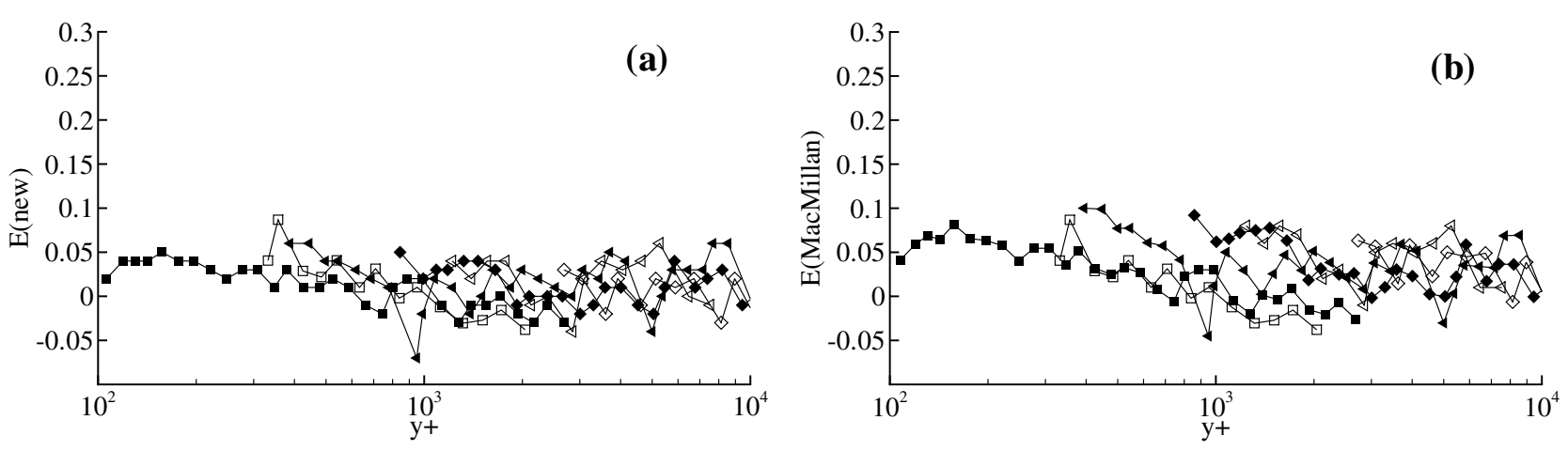

Figure 9. Deviation from the Spalding correlation for selected $R e_{\mathrm{D}} \geqslant 10^{6}$, new (a) and MacMillan (b) corrections with wall terms, points for $y>1 d$ only. Symbols as in figure 6 .
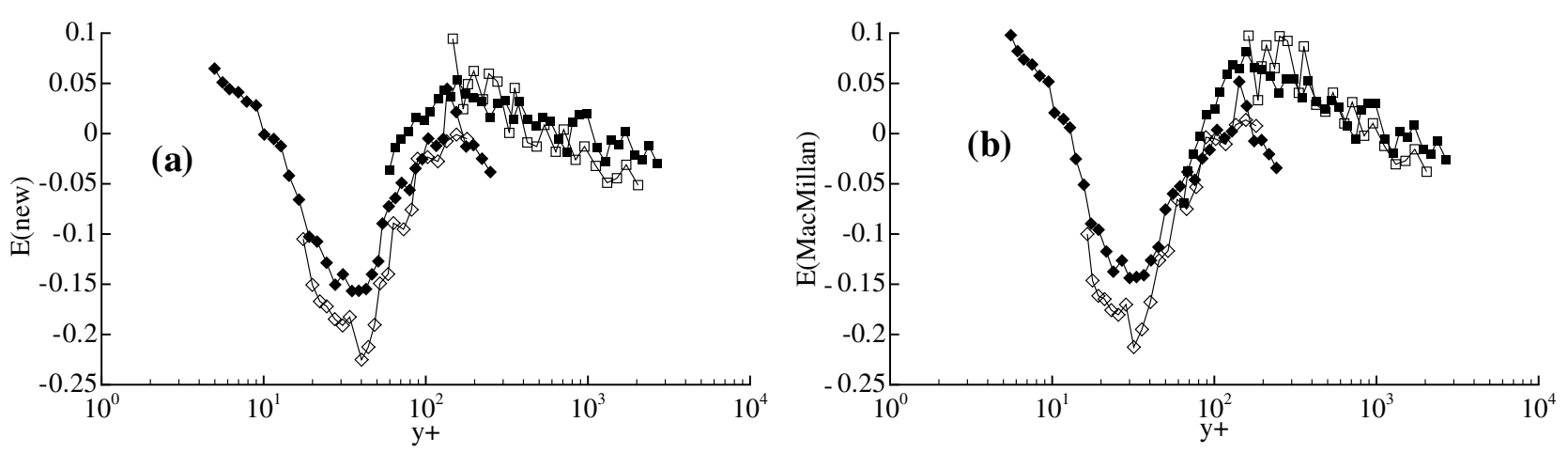

Figure 10. Deviation from Spalding for $R e_{\mathrm{D}}=75 \times 10^{3}$ and $1 \times 10^{6}$, new (a) and MacMillan (b) corrections with wall terms, all data points used. Symbols as in figure 6.

If we wish to use a constant displacement correction, we need to specify a maximum allowed variation in $\alpha$ across the probe, that is, we need to specify the equivalent $y$ position for the first acceptable data point. Based on the examination of the data here, it appears that this point corresponds reasonably well to the point where $y=2 d$.

\subsection{Corrections in the buffer region}

Figure 10 compares the deviation from Spalding's relationship for each correction, using two profiles where the first point is located either in the sublayer $\left(R e_{\mathrm{D}}=75 \times 10^{3}\right)$ or in the overlap region $\left(1 \times 10^{6}\right)$. All the data are shown. The errors close to the wall described above seem to appear only when the point closest to the wall corresponds to approximately $y^{+}>100$. It is apparent that both the MacMillan and the new corrections, with appropriate wall corrections, lead to a good collapse of the profiles in this region of rapidly changing $\alpha$.

The data sets taken with four different probes are used to examine this region further. Figure 11 shows the collapse of the four mean velocity profiles taken at $R e_{\mathrm{D}}=75 \times 10^{3}$ under each correction. The MacMillan and new corrections lead to excellent collapse with diameter and good agreement with the laser Doppler anemometry (LDA) measurements of pipe flow at $R e_{\mathrm{D}}=24600$ by den Toonder and Nieuwstadt (1997). Similar results are observed at $R e_{\mathrm{D}}=150 \times 10^{3}$. Given the experimental uncertainty (estimated at $3.5 \%$ for the point closest to the wall), the 'better' correction of these two in the buffer region cannot be identified with certainty, although the new correction leads to slightly closer agreement to the LDA data.

\section{The true profile in the buffer region}

Can our measurements help to identify the 'true' mean velocity profile near the wall? To consider this matter further, we use data not obtained using Pitot probes.

Figure 12 shows near-wall pipe flow data from LDA (den Toonder and Nieuwstadt 1997), Direct Numerical Simulation (DNS) (Eggels et al 1994) and hot-wire measurements in our pipe flow reported by Morrison et al (2002). den Toonder and Nieuwstadt have shown previously that their LDA measurements in pipe flow (shown here at $R e_{\mathrm{D}}=24600$ ) agree well with $y^{+}=u^{+}$and the DNS of Eggels et al for pipe-flow at a lower $R e_{\mathrm{D}}=5300$ for $y^{+}<20$. Therefore the LDA data of den Toonder and Nieuwstadt (1997) are assumed to be an accurate representation of the true nearwall velocity profile (note that in general, the LDA technique may suffer from its own resolution issues). However, we see that Spalding's relationship diverges from the LDA and DNS results for $20<y^{+}<70$ approximately. In addition, the hotwire data (taken at $R e_{\mathrm{D}}=75 \times 10^{3}$ ) is significantly lower than Spalding's correlation for all $y^{+}$shown.

We look at hot-wire data for the near-wall region in a boundary layer to see if similar trends are observed in figure 13 . Spalding's relationship with $\kappa=0.421$ and $B=5.60$ can be seen once again to misrepresent the velocity profile in the buffer region for $y^{+}>10$ (use of a smaller value of $\kappa$ increases the error). 

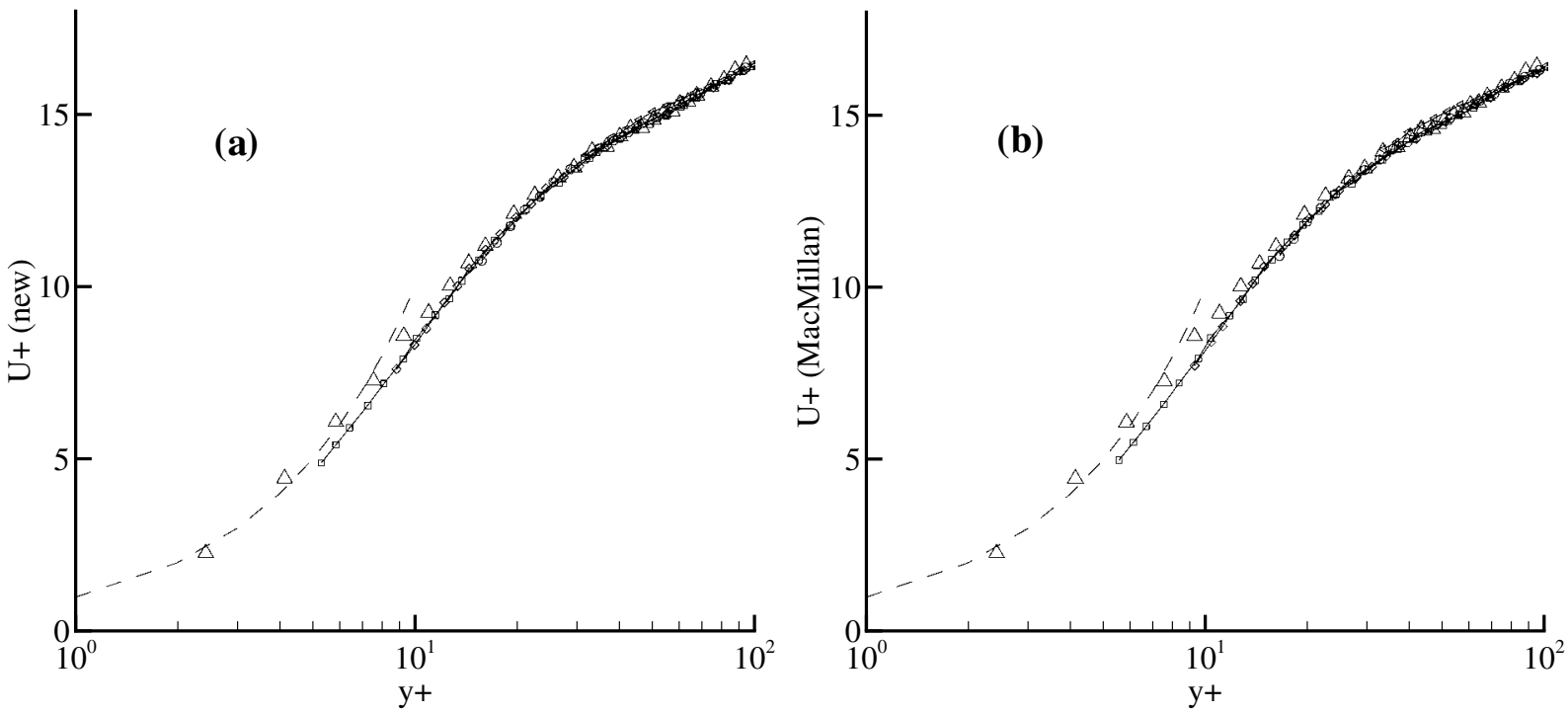

Figure 11. Collapse of mean velocity profiles taken with four different probes, using new (a) and MacMillan (b) corrections, $R e_{\mathrm{D}}=75 \times 10^{3} . \triangle$, LDA data of den Toonder and Nieuwstadt $(1997) ;---, U^{+}=y^{+}$.

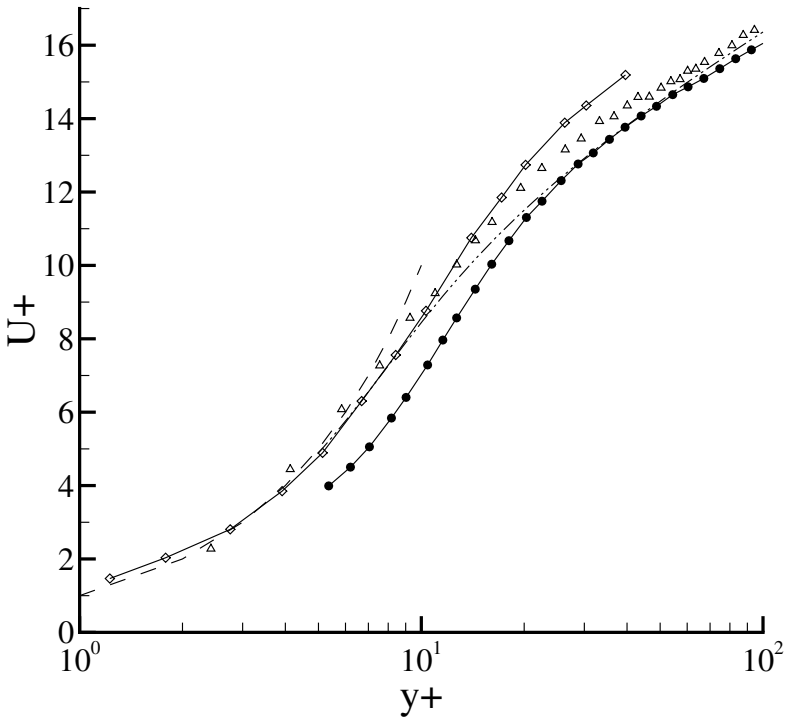

Figure 12. Near-wall pipe flow data taken with means other than a Pitot probe: $\diamond$, DNS data of Eggels et al (1994); $\triangle$, LDA data of den Toonder and Nieuwstadt (1997); $\bullet$, Superpipe hot-wire data by Morrison et al (2002); - - . - Spalding (equation (19)); ,$-- u^{+}=y^{+}$.

To expand the data set, we consider hot-wire measurements in a boundary layer, in particular the subminiature probe data of Ligrani and Bradshaw (1987) and the data of Jones et al (2001), taken at $R e_{\theta}=2620$ and 1800 , respectively (equivalent $R e_{\mathrm{D}} \approx 28 \times 10^{3}$ and $19 \times 10^{3}$ ).

The Ligrani and Bradshaw results blend well into $u^{+}=y^{+}$ near the wall, but the Jones et al measurements suffer from the same problem as the Morrison et al data and throw into doubt the accuracy of data taken near the wall with standard sized hot wires. It can be seen from figure 14 that the hot-wire and Pitot results do not collapse, nor do the hot-wire results approach the LDA profile near the wall: the first 20 or so hot-wire points are too low in each case (apart from the Ligrani and Bradshaw data which agree well with the Pitot results). Jones et al have

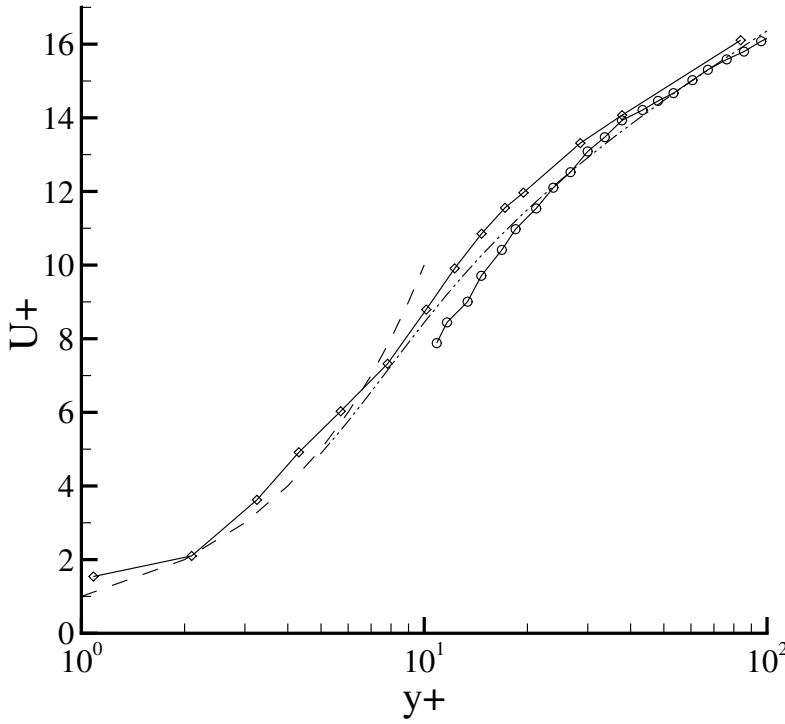

Figure 13. Near-wall boundary layer data taken with means other than a Pitot probe: $O$, Jones et al (2001); $\diamond$, Ligrani and Bradshaw (1987); — - - Spalding (equation (19)); $-\_-, u^{+}=y^{+}$.

suggested that hot-wire measurements provide a standard for judging Pitot tube corrections, but figure 14 shows that their data agree almost exactly with the Superpipe hot-wire data, and both sets of data fall well below the other measurements. Wallconduction effects would increase the measured velocity above the true value rather than below it, and there appears to be some subtlety in acquiring hot-wire data near the wall that has not yet been identified and corrected. Note that this phenomenon is not solely observed at low Reynolds number: the hot-wire velocities near the wall are too low even at $R e_{\mathrm{D}}=3 \times 10^{6}$. This will be a subject of further investigation, but for our present purposes the Superpipe hot-wire data cannot be used as means of establishing the true velocity profile near the wall.

Outside the sublayer, for $y^{+}>30$, there is agreement between all data sets, despite the subtleties of the different 


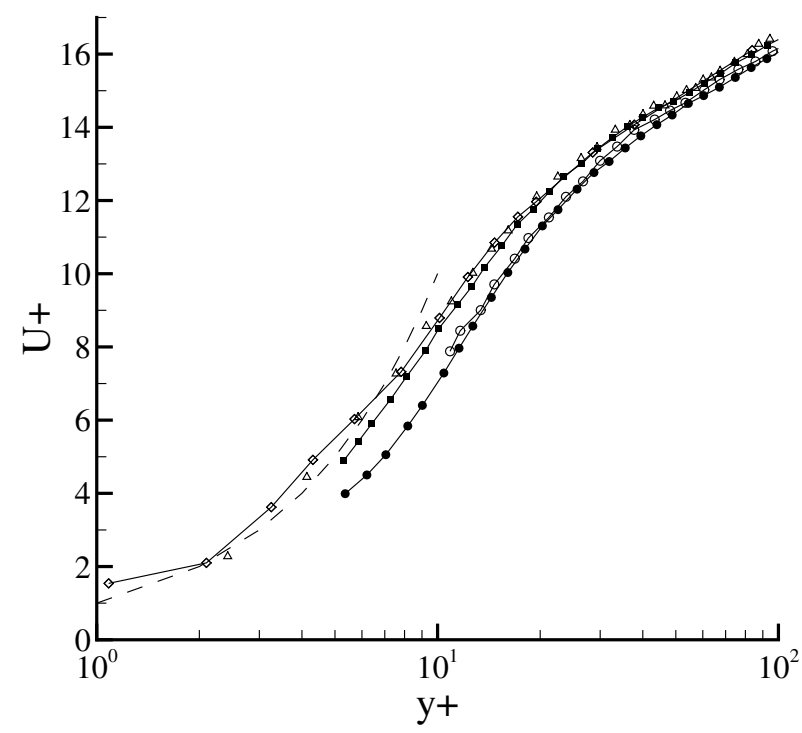

Figure 14. Comparison of near-wall hot-wire and Pitot data in boundary layers and pipes: $\triangle$, LDA data of den den Toonder and Nieuwstadt (1997); $\diamond$, hot-wire data of Ligrani and Bradshaw (1987); O, hot-wire data of Jones et al (2001); $\bullet$, current hot-wire data; $\mathbf{\square}$, current Pitot data (both at $R e_{\mathrm{D}}=75 \times 10^{3}$ ); $-\longrightarrow, u^{+}=y^{+}$.

flows and data acquisition techniques, suggesting that at least for the relatively low Reynolds numbers we can define the 'true' velocity profile for both pipes and boundary layers.

\section{Conclusions}

Displacement and wall corrections have been analysed using data in the range $d^{+}>10$ and $430<D / d<720$. The commonly used displacement corrections due to MacMillan and Chue have been shown not to be accurate for all regions of the flow at all Reynolds numbers. The Chue correction in particular does not collapse data taken with different diameter Pitot probes near the wall.

The new displacement (free shear) correction of equation (10) with the new wall term (equation (17)) has been shown to give excellent collapse in the buffer region at low Reynolds number and in the overlap region for higher Reynolds numbers, for all points with $y>d$ (and $d^{+}>10$ ). The new correction consists of two parts, a displacement term and a wall term. For small $\alpha$ and $y>2 d$, the new correction is identical to the MacMillan correction. The difference between the two then lies in the philosophical point of whether the displacement correction for $\alpha=0$ must be equal to zero (new) or not (MacMillan). For large $\alpha$ and $y<2 d$, the new wall term has been shown to give more reliable collapse with diameter closer to the wall than the MacMillan correction. This wall term appears to be the key component in support of the new corrections proposed here. It is recommended that data for $y<d$ are discarded if the new correction is used, and that data for $y<2 d$ are discarded if the MacMillan correction is used.

If points where $y<2 d$ are removed (that is, if the need for a wall term is avoided) and either the MacMillan or new corrections are used, almost identical results are obtained in the overlap region, that is, the results in the overlap region are independent of displacement correction.
We have also noted significant discrepancies between corrected Pitot probe mean velocity profiles and those obtained using single normal hot wires, although the miniature hot wire of Ligrani and Bradshaw gave good agreement with the Pitot probe results. Further work on the performance of hot wires close to a wall is required before hot wires can be used with confidence to measure near-wall velocity profiles. Certainly, hot-wire data should not be used as the standard to which data obtained using other methods should be compared.

\section{Acknowledgments}

The support of ONR under grant nos N00014-98-1-0325 and N00014-99-1-0340, and NSF under grant CTS-9908442 is gratefully acknowledged. JFM is indebted to both the Engineering and Physical Sciences Research Council (GR/M64536/01) and the Royal Academy of Engineering (England) for financial support.

\section{References}

Barenblatt G I and Chorin A J 1998 Scaling of the intermediate region in wall-bounded turbulence: the power law Phys. Fluids 101043

Barker M 1922 On the use of very small Pitot-tubes for measuring wind velocities Proc. R. Soc. A $101435-45$

Chue S H 1975 Pressure probes for fluid measurement Prog. Aerosp. Sci. 16 147-223

den Toonder J M J and Nieuwstadt F T M 1997 Reynolds number effects in a turbulent pipe flow for low to moderate Re Phys. Fluids 93398

Dickinson J 1975 Turbulent skin friction techniques Internal Report Laval University, Quebec

Eggels J G M, Unger F, Weiss M H, Westerweel J, Adrian R J, Friedrich R and Nieuwstadt F T M 1994 Fully developed turbulent pipe flow: a comparison between direct numerical simulation and experiment J. Fluid Mech. 268 175-209

Hall I M 1956 The displacement effect of a sphere in two-dimensional shear flow J. Fluid Mech. 1 142-62

Hurd C W, Chesky K P and Shapiro A H 1953 Influence of viscous effects on impact tubes J. Appl. Mech. 248 253-6

Jones M B, Marusic I and Perry A E 2001 Streamwise evolution and structure of sink-flow turbulent boundary layers J. Fluid Mech. 428 1-27

Lighthill M J 1957 Contributions to the theory of the Pitot-tube displacement effect J. Fluid Mech. 1 493-512

Ligrani P M and Bradshaw P 1987 Spatial resolution and measurement of turbulence in the viscous sublayer using subminiature hot-wire probes Exp. Fluids 5 407-17

Livesey H J L 1956 The behavior of transverse cylindrical and forward facing total pressure probes in transverse total pressure gradient J. Basic Eng. 23 949-55

MacMillan F A 1954 Viscous effects on Pitot tubes at low speeds J. R. Aero. Soc. 58 837-9

MacMillan F A 1956 Experiments on Pitot tubes in shear flow Ministry of Supply, Aero. Res. Council R \& M 3028

McKeon B J, Morrison J F, Jiang W, Li J and Smits A J 2003 Revised log law constants for fully-developed turbulent pipe flow Reynolds Number Scaling in Turbulent Flow: Proc. IUTAM Symp. ed A J Smits (Dordrecht: Kluwer)

McKeon B J and Smits A J 2002 Static pressure correction in high Reynolds number fully developed turbulent pipe flow Meas. Sci. Technol. 13 1608-14

Morrison J F, Jiang W, McKeon B J and Smits A J 2002 Reynolds-number dependence of streamwise velocity statistics in turbulent pipe flow AIAA Paper 2002-0574

Ozarapoglu V 1972 PhD Thesis Laval University, Quebec 
Patel V C 1965 Calibration of the Preston tube and limitations on its use in pressure gradients J. Fluid Mech. 23 185-208

Perry A E, Hafez S and Chong M S 2001 A possible reinterpretation of the Princeton Superpipe data J. Fluid Mech. 439 395-401

Spalding D B 1961 A single formula for the law of the wall Trans. ASME E 28 455-8

Tavoularis S and Szymczak M 1989 Displacement effects of square-ended Pitot tubes in shear flows Exp. Fluids 7 33-7
Young A D and Maas J N 1936 The behaviour of a Pitot-tube in a transverse total-pressure gradient Aero. Res. Council $R \& M$ $\mathbf{1 7 7 0}$

Zagarola M V 1996 Mean flow scaling in turbulent pipe flow PhD Thesis Princeton University

Zagarola M V and Smits A J 1998 Mean-flow scaling of turbulent pipe flow J. Fluid Mech. 373 33-79 